\title{
Constraints in antigen presentation severely restrict $T$ cell recognition of the allogeneic fetus
}

\author{
Adrian Erlebacher, ${ }^{1}$ Daniela Vencato, ${ }^{1}$ Kelly A. Price, ${ }^{2}$ Dorothy $Z$ hang, ${ }^{2}$ and Laurie H. Glimcher ${ }^{2,3}$ \\ 1Department of Pathology, Experimental Pathology Program, New York University School of Medicine, New York, New York, USA. \\ 2Department of Immunology and Infectious Diseases, Harvard School of Public Health, Boston, Massachusetts, USA. \\ ${ }^{3}$ Department of Medicine, Harvard Medical School, Boston, Massachusetts, USA.
}

\begin{abstract}
How the fetus escapes rejection by the maternal immune system remains one of the major unsolved questions in transplantation immunology. Using a system to visualize both $\mathrm{CD}^{+}$and $\mathrm{CD8}^{+} \mathrm{T}$ cell responses during pregnancy in mice, we find that maternal $T$ cells become aware of the fetal allograft exclusively through "indirect" antigen presentation, meaning that $T$ cell engagement requires the uptake and processing of fetal/placental antigen by maternal APCs. This reliance on a relatively minor allorecognition pathway removes a major threat to fetal survival, since it avoids engaging the large number of $T$ cells that typically drive acute transplant rejection through their ability to directly interact with foreign $\mathrm{MHC}$ molecules. Furthermore, $\mathrm{CD8}^{+} \mathrm{T}^{\mathrm{c}}$ cells that indirectly recognize fetal/placental antigen undergo clonal deletion without priming for cytotoxic effector function and cannot induce antigen-specific fetal demise even when artificially activated. Antigen presentation commenced only at mid-gestation, in association with the endovascular invasion of placental trophoblasts and the hematogenous release of placental debris. Our results suggest that limited pathways of antigen presentation, in conjunction with tandem mechanisms of immune evasion, contribute to the unique immunological status of the fetus. The pronounced degree of $T$ cell ignorance of the fetus also has implications for the pathophysiology of immune-mediated early pregnancy loss.
\end{abstract}

\section{Introduction}

The distinction between "direct" and "indirect" antigen presentation provides an important framework for understanding afferent mechanisms in transplant rejection (1). In the direct pathway, host $\mathrm{T}$ cells directly engage foreign peptide/ $\mathrm{MHC}$ complexes on the surface of donor cells. In the indirect pathway, they engage donorderived peptides presented, or cross-presented, by host APCs in a host MHC-restricted fashion. Due to their high frequency within the peripheral $\mathrm{T}$ cell repertoire, directly alloreactive $\mathrm{T}$ cells have been largely considered the major acute threat to graft survival (1-3). Indeed, organs such as the heart show poor or absent rejection when only indirect antigen presentation is available (4-8).

Direct and indirect allorecognition pathways have not been studied during pregnancy, a unique situation where an allogeneic tissue escapes rejection despite extensive contact with the host $(9,10)$. Furthermore, the fate of maternal $\mathrm{T}$ cells responding to fetal/placental antigens by either allorecognition pathway remains largely unknown. These issues are poorly understood in large part because it is unclear how extensively maternal $\mathrm{T}$ cells are even aware of fetal alloantigens. To date, there has been no report to our knowledge of maternal $\mathrm{CD}^{+} \mathrm{T}$ cell recognition of the fetus, and conflicting data have emerged regarding maternal $\mathrm{CD}^{+} \mathrm{T}$ cell responses during pregnancy. In one study, dramatic phenotypic changes were observed as early as E9.5 in splenic CD8 ${ }^{+}$ T cells expressing a transgenic TCR directly alloreactive with paternal MHC class I (11). This response, however, has been difficult to

Nonstandard abbreviations used: B6, C57BL/6; CD62L, CD62 ligand; FDC, follicular dendritic cell.

Conflict of interest: The authors have declared that no conflict of interest exists. Citation for this article: J. Clin. Invest. 117:1399-1411 (2007). doi:10.1172/JCI28214. reconcile with the very low levels of MHC class I (both heavy chain and $\beta 2$-microglobulin) expressed by the murine conceptus during the first half of gestation (12-14). Indeed, 2 other studies using TCR transgenic mice with different anti-MHC class I TCR clonotypes showed minor or absent phenotypic changes in maternal $\mathrm{CD}^{+} \mathrm{T}$ cells $(15,16)$, which instead suggests immune ignorance. Antigen-specific maternal $\mathrm{T}$ cell responses to minor histocompatibility antigens have only been demonstrated in the context of TCR transgenic mice bearing $\mathrm{CD}^{+} \mathrm{T}$ cells specific for male $\mathrm{H}-\mathrm{Y}$ antigen, and only in late gestation (17). Critically, all of these studies relied upon the use of TCR transgenic females as mating partners, which might have led to nonphysiological or absent $\mathrm{T}$ cell responses (18), and none of them directly visualized $\mathrm{T}$ cell proliferation, perhaps the best indication of actual TCR engagement.

Recently, a report that took the alternative approach of $\mathrm{T}$ cell adoptive transfers found no response in antipaternal MHC class I and anti-H-Y TCR transgenic T cells when pregnant mice were observed up until E6.5, 3 days after implantation (19). However, to our knowledge, adoptive transfers have not been used to study maternal $\mathrm{T}$ cell responses during the main period of gestation. This approach provides a physiological context for visualizing $T$ cell activation and can be used to finely dissect the anatomical sites and temporal onset of antigen presentation. This latter issue is of interest since several models of allospecific abortion in mice show fetal demise relatively early in gestation (i.e., E9.5-E13.5), despite the lack of documented antigen-specific $\mathrm{T}$ cell activation (20-23).

Here, we study fetal/placental antigen presentation by combining $T$ cell adoptive transfers with use of the Act-mOVA transgenic mouse line recently generated by Ehst et al. (24). These mice express a membrane-bound form of OVA from the ubiquitously active 

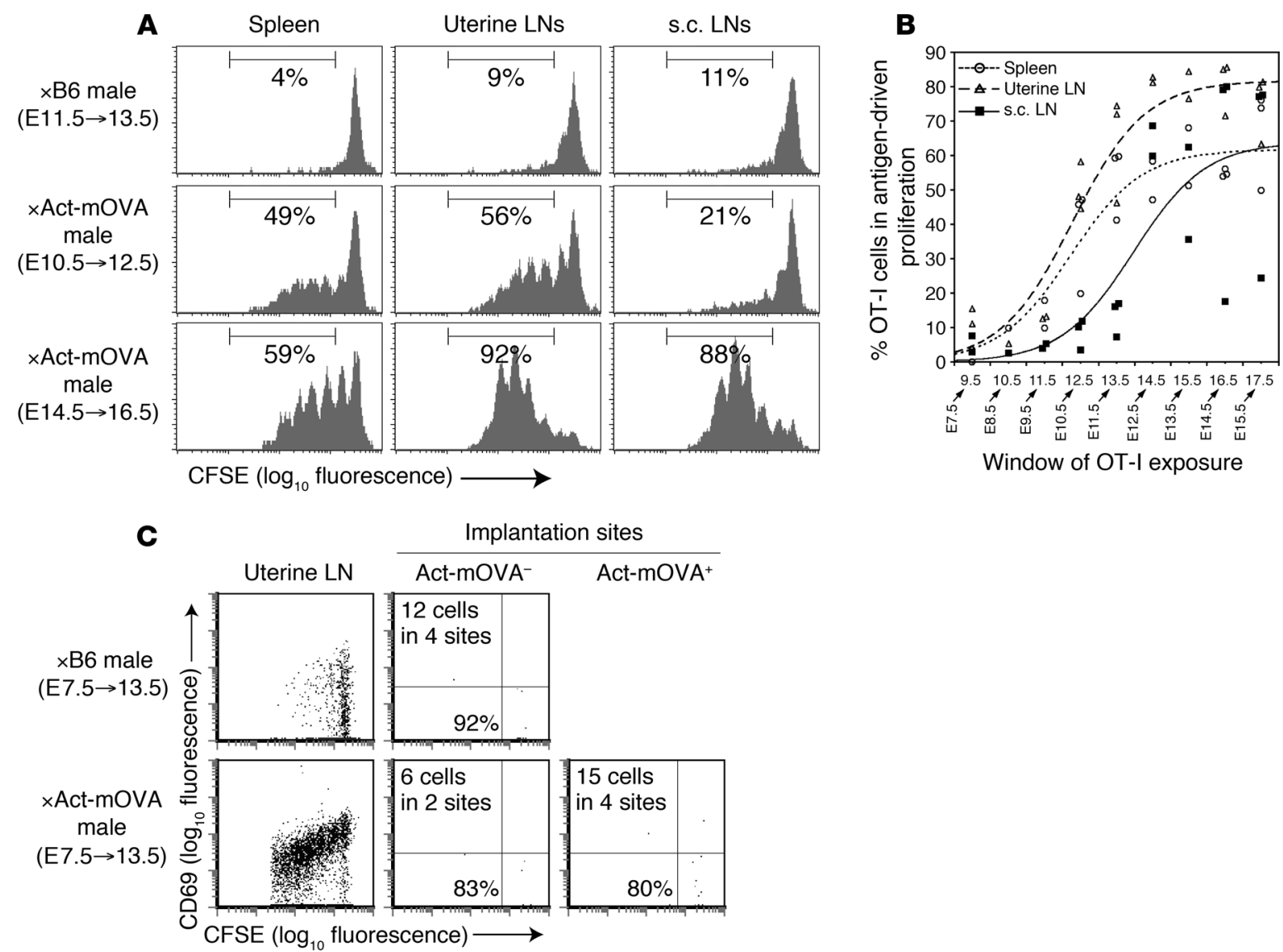

\section{Figure 1}

Spatiotemporal patterns of OT-I T cell recognition of fetal/placental OVA. B6CBAF1 females mated to Act-mOVA or B6 control males were adoptively transferred with CFSE-labeled B6CBAF1 OT-I T cells. (A) OT-I proliferation in secondary lymphoid organs. OT-I cells were visualized by flow cytometry at the end of their indicated 2-day window of exposure. The percentage of cells undergoing more than 1 cell division is noted; such cells were only rarely observed in B6-mated females at any stage of gestation. (B) Midgestational onset of OT-I proliferation in secondary Iymphoid organs. Act-mOVA-mated female mice at different stages of gestation were transferred with OT-I cells and then sacrificed 2 days later. Using raw values for proliferation determined as in $\mathbf{A}$, the percentage of OT-I cells in antigen-driven proliferation was calculated by subtracting mean B6-mated control values for each of the 3 secondary lymphoid organ sets in a given experiment from their respective values in individual Act-mOVA-mated mice. The graph shows all data for $n=21$ Act-mOVA-mated females over 5 independent experiments. (C) Absence of OT-I reactivity at the maternal/fetal interface in early gestation. Transferred OT-I cells were visualized in the uterine LNs and in dissected decidua/placentas pooled from several implantation sites. Many of these cells lie along the $x$ axis of the plot and so are difficult to see. We therefore note the total number of OT-I cells recovered from these sites. Percentages indicate the fraction of OT-I cells with a CFSEundiluted CD69lo naive phenotype (cells in the lower-right quadrant).

$\beta$-actin promoter and were originally used in experiments designed to visualize the priming of $\mathrm{CD}^{+}$and $\mathrm{CD}^{+} \mathrm{T}$ cells in response to skin grafts in which the only histocompatibility antigen expressed differently between donor and recipient was OVA (24). In our system, we directed OVA expression exclusively to the fetal allograft by mating Act-mOVA transgenic males to nontransgenic females. We then adoptively transferred $\mathrm{CD} 4^{+}$or $\mathrm{CD} 8^{+} \mathrm{TCR}$ transgenic $\mathrm{T}$ cells specific for OVA-derived peptides and monitored their responses during pregnancy. Use of this system has allowed us to determine the spatial and temporal patterns of antigen dissemination from the murine conceptus and to study the behavior of $\mathrm{T}$ cells reactive with a model fetal/placental antigen. We demonstrate that recognition of the fetus occurs exclusively via indirect allorecognition, which leaves the population of T cells posing the greatest threat to transplant survival entirely ignorant of the fetal allograft.

\section{Results}

Spatial and temporal patterns of $\mathrm{CD} 8^{+} T$ cell recognition of fetal/placental OVA. Our initial experiments focused on defining the spatial and temporal availability of fetal/placental OVA for presentation to maternal $\mathrm{T}$ cells over the course of gestation. To this end, we used $\mathrm{CD}^{+} \mathrm{T}$ cells isolated from OT-I TCR transgenic mice. These cells express a transgenic TCR specific for the $\mathrm{OVA}_{257-264}$ epitope SIINFEKL presented by the MHC class I molecule $\mathrm{H}-2 \mathrm{~K}^{\mathrm{b}}$ and undergo prompt and synchronous cell division when appropriately stimulated (25). To track the cells in vivo, we labeled them with the fluorescent dye CFSE, which also allows each cell division to be visualized by flow cytometry as a sequential 2-fold reduction in fluorescence intensity. The cells were then transferred into wildtype female mice impregnated by males hemizygous for the ActmOVA transgene, thus exposing them to a maternal host with a litter half consisting of Act-mOVA ${ }^{+}$concepti. Two days later, the mice 

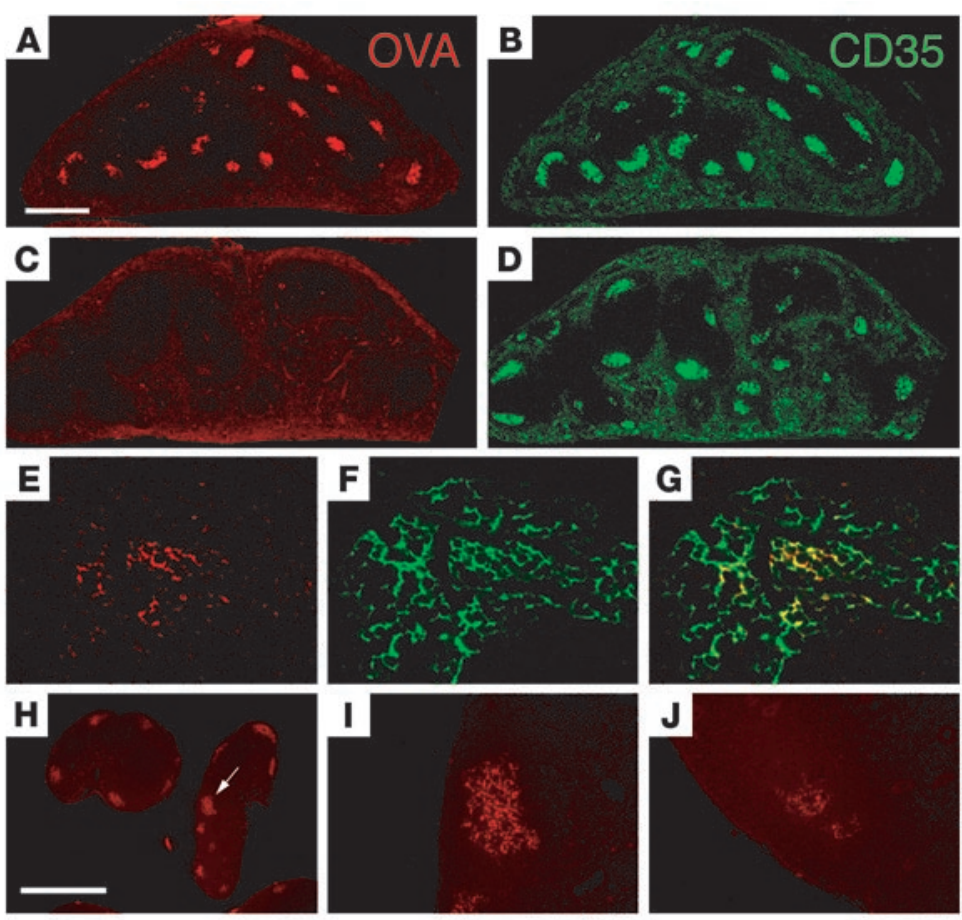

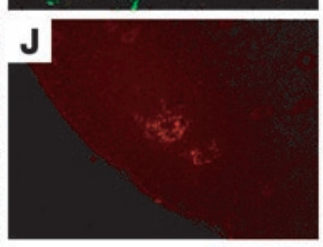

\section{Figure 2}

OVA shed from the conceptus accumulates on FDCs. (A-G) OVA accumulation on splenic FDCs. (A-D) E17.5 spleens from Act-mOVA-mated (A and $\mathbf{B}$ ) or B6-mated (C and $\mathbf{D})$ mice double stained for OVA (red; $\mathbf{A}$ and $\mathbf{C}$ ) and CD35 (green; $\mathbf{B}$ and $\mathbf{D}$ ). Red pulp is apparent as areas of diffuse green staining resulting from rbc rendered artifactually fluorescent by the immunohistochemistry protocol. Data are representative of $n=8-9$ mice per group on E15.5-E17.5. Scale bar: $0.5 \mathrm{~mm}$. (E-G) High-power confocal photomicrographs of a double-stained OVA+ focus, showing OVA staining (E), CD35 staining (F), and the merged image (G). (H-J) OVA accumulation on LN FDCs. (H) Two subcutaneous LNs from an ActmOVA-mated mouse on E17.5; a higher magnification of the $\mathrm{OVA}^{+}$focus indicated with an arrow is shown in I. Data are representative of all $n=8$ mice analyzed at E15.5-E17.5. (J) An OVA+ focus in a uterine LN on E11.5, as such foci begin to appear. Similar foci were seen in $n=3$ of 4 mice analyzed. Scale bar: $1 \mathrm{~mm}(\mathbf{H})$ and $0.2 \mathrm{~mm}$ in (I and $\mathbf{J})$. As in $\mathbf{C}$, LNs from B6-mated mice showed no OVA+ foci (data not shown).

were sacrificed and the transferred cells were analyzed. The short time period of 2 days was chosen in order to minimize lymphocyte recirculation and thus capture snapshots of the distribution of OVA at various anatomical locations during defined windows of gestation. As negative controls, we performed parallel adoptive transfers of OT-I cells into females mated to C57BL/6 (B6) males ( $\mathrm{H}-2^{\mathrm{b}}$ haplotype), the background strain of the Act-mOVA line. In order to maximize pregnancy rates, we used highly fertile B6CBAF1 females ( $\mathrm{H}-2^{\mathrm{b} / \mathrm{k}}$ haplotype) as mating partners (26). This necessitated generating OT-I mice on a B6CBAF1 background as source of cells for adoptive transfer.

As shown in Figure 1A, OT-I T cells proliferated in the secondary lymphoid organs of female mice mated to Act-mOVA males (middle and bottom rows) but not in females mated to control B6 males (top row). By progressively sampling 2-day gestational windows (Figure 1B), we found that vigorous proliferation with up to 5 rounds of division appeared abruptly and consistently in the spleen and draining LNs of the uterus (the paraaortic and renal nodes, referred to hereafter as the uterine LNs) on E12.5, 2 days after transfer on E10.5 (e.g., Figure 1A, middle row). Since naive OT-I cells initiate proliferation only 24 hours after initial antigen exposure and then have a cell cycle time of about 5 hours (27), this timing implied that substantial amounts of OVA first became available for presentation within secondary lymphoid organs around E10.5. By E14.5, OT-I proliferation also variably appeared in the subcutaneous LNs (Figure 1A, bottom row) and mesenteric LNs (data not shown). We additionally determined the CFSE dilution profile of OT-I cells within the pooled decidua and placentas of implantation sites themselves, sampling gestational windows from E4.5-E10.5 to E7.5-E13.5. As shown in Figure 1C, proliferating OT-I cells were not observed in implantation sites containing $\mathrm{OVA}^{+}$concepti as late as E13.5, despite vigorous proliferation in the uterine $\mathrm{LN}$, nor was there cell-surface upregulation of CD69, a very early activation marker that shows increased expression upon TCR engagement even prior to cell division. OT-I cells were furthermore generally very rare within implantation sites $(<5$ cells per implantation site per million cells injected; data not shown), and despite the difficulty of a statistical analysis there were no obvious differences in number between those containing $\mathrm{OVA}^{+}$and $\mathrm{OVA}^{-}$concepti. Together, these data demonstrated that the secondary lymphoid organs were the primary sites of OVA presentation to maternal $\mathrm{T}$ cells and that presentation only started at mid-gestation $(\sim \mathrm{E} 10.5)$.

We next sought independent confirmation that the disparate sites of OT-I proliferation truly reflected the local presence of antigen. Strikingly, anti-OVA immunohistochemistry performed on spleens of Act-mOVA-mated females on or after E12.5 revealed distinct foci of OVA immunoreactivity confined to areas staining strongly positive for the follicular dendritic cell (FDC) marker CD35 (complement receptor CR1; Figure 2, A-D). Although closely associated on individual cells, OVA immunoreactivity (red) was frequently broader than CD35 immunoreactivity (green), suggesting OVA accumulation on the FDC surface (Figure 2, E-G). Since the spleen does not receive any lymphatic drainage, the presence of immunoreactive OVA in this organ directly demonstrates OVA release from the conceptus into the maternal circulation. $\mathrm{OVA}^{+}$ foci also first consistently appeared in the uterine and subcutaneous LNs on E11.5 and E15.5, respectively (Figure 2, H-J).

Further studies suggested that the placenta was the main source of shed antigen. Immunohistochemistry performed on Act-mOVA concepti on E7.5, about 3 days prior to the onset of antigen presentation, revealed high OVA expression mainly in a population of trophoblasts at the invasive front of the ectoplacental cone (Supplemental Figure 1; supplemental material available online with this article; doi:10.1172/JCI28214DS1). These cells continued to express high levels of OVA on E9.5 (Figure 3, C and D), at which time we additionally detected high expression in trophoblast giant cells surrounding the entire conceptus (Figure 3, A and B) and low expression in the embryo proper and the body of the 

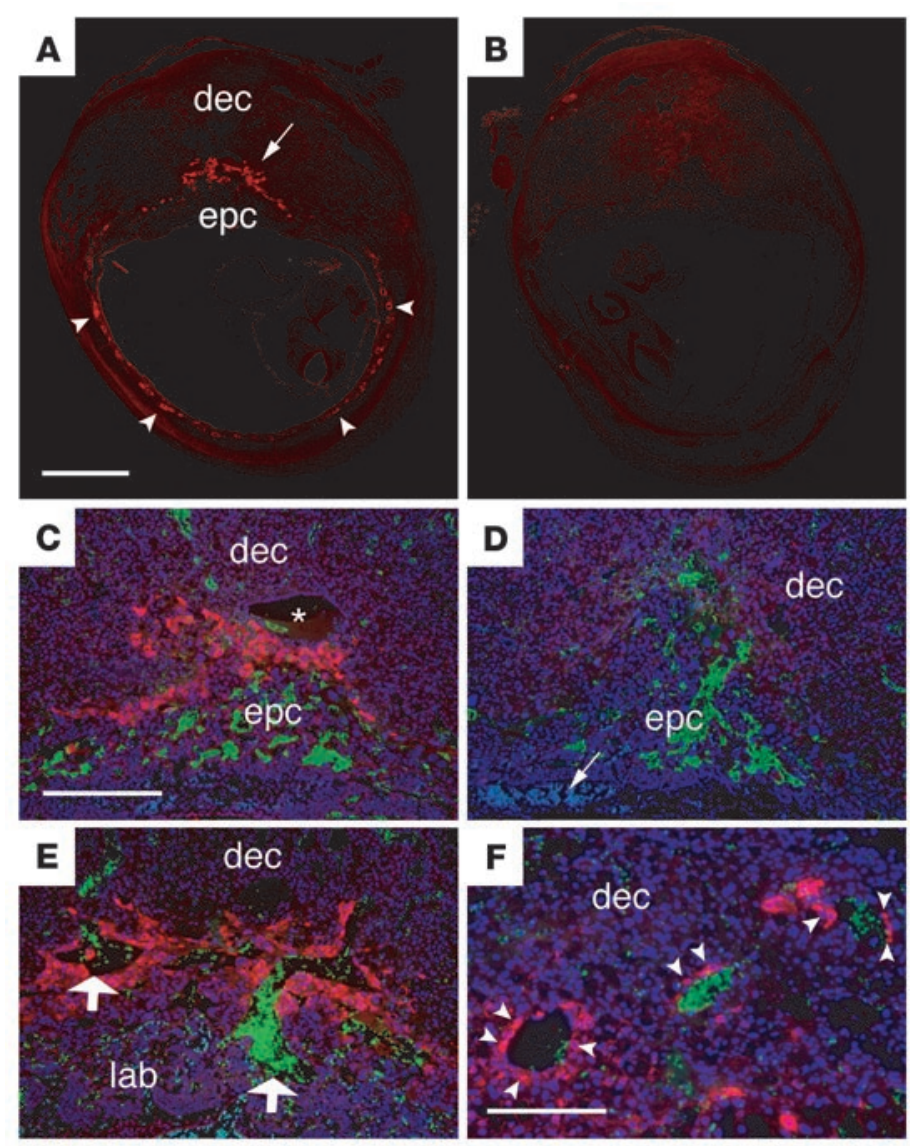

\section{Figure 3}

Act-mOVA expression at the maternal/fetal interface. Sections of implantation sites of Act-mOVA (A, C, E, and F) or nontransgenic concepti (B and $\mathbf{D}$ ) stained with anti-OVA antibodies (red). C-F also show artifactually fluorescent maternal red blood cells (green) to demarcate maternal blood vessels, and DAPI-stained nuclei (blue). (A and B) E9.5 implantation sites show high OVA expression in transgenic concepti $(n=9)$ confined to trophoblast giant cells (arrowheads) and trophoblasts at the invasive front of the ectoplacental cone (epc; arrow) abutting the maternal decidua (dec). Scale bar: $1 \mathrm{~mm}$. (C-E) Higher-power magnifications show that transgenic trophoblasts expressing high levels of OVA had not yet invaded maternal arteries by E9.5 (C and $\mathbf{D})$ but were lining maternal vessels (arrows) by E10.5 $(\mathbf{E} ; n=5)$. Transgenic trophoblasts expressed low levels of OVA in the body of the ectoplacental cone and developing labyrinth (lab), where they also contact maternal blood (compare the purple-tinged color of these structures in $\mathbf{C}$ and $\mathbf{E}$, reflecting a combination of OVA staining and the DAPI counterstain, with their blue color in D). Fetal rbc appear blue-tinged due to their nucleation (arrow in D); the asterisk in $\mathbf{C}$ marks the vestigial uterine lumen. Scale bar: $0.4 \mathrm{~mm}$. (F) At E13.5, transgenic trophoblasts expressing high levels of OVA were closely associated with or lining (arrowheads) maternal arteries deeper in the decidua $(n=3)$. By late gestation, high OVA expression was also apparent in the spongiotrophoblast layer (data not shown). Scale bar: $0.2 \mathrm{~mm}$. developing placenta (Figure 3, A-D, and data not shown). On E9.5, OVA $^{\text {hi }}$ ectoplacental trophoblasts had only just begun to appear within maternal arterioles as part of the normal developmental process of endovascular invasion (Figure 3, C and D). By E10.5, however, these cells were clearly lining maternal blood vessels (Figure $3 \mathrm{E}$ ), and their high OVA expression levels persisted later into gestation (Figure 3F). Endovascular invasion thus coincided with our estimate for the initial presentation of OVA to OT-I cells in the spleen on E10.5. Importantly, intravenous injections of up to 2 spleens' worth of whole splenocytes (both rbc and leukocytes; about $2 \times 10^{6}$ cells) from Act-mOVA late-gestational embryos or neonates into B6-mated females did not lead to OVA accumulation on FDCs (Supplemental Figure 2). Similarly, the intravenous injection of $1 \mathrm{mg}$ soluble OVA into B6-mated females did not lead to FDC accumulation (Supplemental Figure 2).

Maternal $C D 8^{+} T$ cells recognize fetal/placental OVA exclusively via the indirect allorecognition pathway. The physical presence of OVA in all secondary lymphoid organs strongly suggested that OT-I proliferation was largely induced by maternal APCs that had locally ingested antigen and cross-presented OVA peptide. Such presentation would be categorized as "indirect." However, it remained possible that OT-I proliferation was also induced by antigen presentation by migratory fetal cells expressing OVA or by the hematogenous shedding of OVA peptide/MHC class I complexes from the conceptus and their subsequent uptake and retention as intact molecules on the cell surface of maternal APCs. These modes of presentation would be included in the categories of the classical "direct" pathway or the recently described "semidirect" pathway (28), respectively.
To distinguish between these possibilities, we established a mating scenario that allowed us to determine whether OVA presentation to OT-I cells in Act-mOVA-mated females still occurred when only the conceptus expressed the required MHC restriction element. These experiments took advantage of B6.C- $H 2^{b m 1}$ mice, which are $\mathrm{B} 6$ congenics that express a mutant $\mathrm{H}-2 \mathrm{~K}^{\mathrm{b}}$ molecule unable to present SIINFEKL peptide (29). We first crossed B6.C-H2 $2^{b m 1}$ and CBA $\left(\mathrm{H}-2^{\mathrm{k}}\right.$ haplotype $)$ mice to generate $(\mathrm{bm} 1 \times \mathrm{CBA}) \mathrm{F}_{1}$ hybrid females. These females express $\mathrm{H}-2 \mathrm{~K}^{\mathrm{bm} 1}$ and $\mathrm{H}-2 \mathrm{~K}^{\mathrm{k}}$ and so are also unable to present OVA to OT-I cells, yet they otherwise parallel the B6CBAF1 females used in our initial experiments. Due to their MHC disparity, the B6CBAF1 OT-I cells used in the experiments described above were partially rejected in $(\mathrm{bm} 1 \times \mathrm{CBA}) \mathrm{F}_{1}$ mice after 2 days, leading to a 2 - to 3-fold decrease in cell number (data not shown). Nonetheless, this alloresponse did not preclude using them to detect OVA presentation, since they showed the same CFSE dilution profile in $\mathrm{B} 6 \mathrm{CBAF} 1$ and $(\mathrm{bm} 1 \times \mathrm{CBA}) \mathrm{F}_{1}$ hosts when the mice were given SIINFEKL-pulsed B6CBAF1 splenocytes as a source of $\mathrm{K}^{\mathrm{b}} / \mathrm{SIINFEKL}$ complexes (Figure 4A).

To determine whether $\mathrm{H}-2 \mathrm{~K}^{\mathrm{b}}$ expression by maternal APCs was required for fetal/placental OVA presentation, we transferred OT-I cells into Act-mOVA-mated (bm1 1 CBA) $\mathrm{F}_{1}$ females on or after E12.5. Since Act-mOVA males are homozygous for $H-2 K^{b}$, all concepti still bore $1 \mathrm{H}-2 \mathrm{~K}^{b}$ allele and thus expressed the necessary MHC determinant for OT-I activation. Nonetheless, the transferred cells failed to proliferate and showed the same CFSE dilution profile as when transferred into B6-mated females (Figure 4B). This stood in contrast to the robust and consistent proliferation seen in Act- 
A

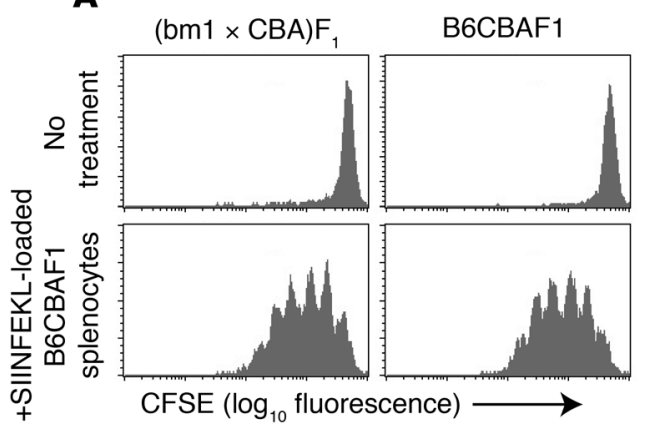

Figure 4

\section{Indirect prese}

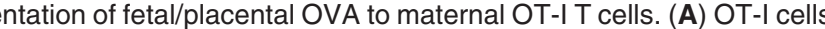
on a B6CBAF1 background are able to detect $\mathrm{K}^{\mathrm{b}} / \mathrm{SIINFEKL}$ complexes in (bm1 $\times \mathrm{CBA}) \mathrm{F}_{1}$ mice. Splenocytes from $(\mathrm{bm} 1 \times \mathrm{CBA}) \mathrm{F}_{1}$ or $\mathrm{B} 6 \mathrm{CBAF} 1$ female virgins were analyzed 2 days after the injection of CFSE-labeled B6CBAF1 OT-I cells, with or without a concurrent injection of SIINFEKL-loaded B6CBAF1 splenocytes. Histograms were normalized to account for the loss of OT-I cells in $(\mathrm{bm} 1 \times \mathrm{CBA}) \mathrm{F}_{1}$ mice. (B) Lack of OT-I proliferation in $(\mathrm{bm} 1 \times \mathrm{CBA}) \mathrm{F}_{1}$ females mated to Act-mOVA males. Splenocytes from B6- or Act-mOVA-mated $\left(\mathrm{bm} 1 \times \mathrm{CBA} \mathrm{F}_{1}\right.$ females were analyzed 2 days after the injection of CFSE-labeled B6CBAF1 OT-I cells on or after E12.5. Data are representative of $n=10$ Act-mOVA-mated $(\mathrm{bm} 1 \times \mathrm{CBA}) \mathrm{F}_{1}$ and $n=3$ B6-mated mice in 3 independent experiments. Identical results were obtained with LN cells.

mOVA-mated B6CBAF1 females (Figure 1, A and B). Thus, we conclude that OVA presentation to maternal OT-I cells does not involve the direct engagement of fetal or placental peptide/MHC class I complexes and is instead mediated exclusively by maternal APCs and indirect presentation.

Directly alloreactive $T$ cells remain ignorant of paternal $H-2 K$ over the entirety of gestation. Our unambiguous demonstration of indirect fetal/placental OVA presentation stood in stark contrast to previous contradictory findings regarding the ability of $\mathrm{CD}^{+} \mathrm{T}$ cells to directly recognize paternal MHC class I molecules during pregnancy $(11,15,16)$. In particular, our data would not have been predicted by a report that antipaternal MHC class I TCR transgenic T cells show dramatic phenotypic changes as early as E9.5 in intact TCR transgenic mice (11). However, this report is inconsistent with data suggesting that the conceptus does not express MHC class I in the first half of gestation (12-14). Indeed, we confirmed these latter findings by performing anti-MHC class I immunohistochemistry on implantation sites from wild-type and MHC class I-deficient mice ( $H 2 K^{b} D^{b}$ knockout mice) mated in various combinations to selectively allow for MHC class I expression in the maternal and fetal compartments together, the fetal compartment alone, or neither compartment (Supplemental Figure 3).

To understand these discrepant observations, we thoroughly and directly assessed the level of direct recognition of paternal $\mathrm{MHC}$ class I molecules during pregnancy using an adoptive transfer model that paralleled our OT-I transfer experiments and that avoided the potential pitfalls of using intact TCR transgenic mice as mating partners. Specifically, we used $\mathrm{CD}^{+} \mathrm{T}$ cells isolated from BM3 TCR transgenic mice (CBA strain background $\left[\mathrm{H}-2^{\mathrm{k}}\right.$ haplotype]) (30). These cells express a transgenic TCR that shows direct alloreactivity with $\mathrm{H}-2 \mathrm{~K}^{\mathrm{b}}$ itself (30). The cells were CFSE labeled and transferred into CBA females impregnated by either syngeneic CBA or allogeneic B6 (H-2 $\left.{ }^{b}\right)$ males. In both situations, the BM3 cells remained naive, in that they did not proliferate in the spleen, venous injection of B6CBAF1 splenocytes, indicating that there was no differential loss in responsiveness in these cells to exogenous $\mathrm{H}-2 \mathrm{~K}^{\mathrm{b}}$ molecules (Figure 5 and Supplemental Figure 4). Last, BM3 proliferation was undetectable in the secondary lymphoid organs of females mated to Act-mOVA males (data not shown $[n=5]$; we did not assess implantation sites or activation marker expression profiles with these crosses). Together, these results strongly suggested that BM3 cells are simply ignorant of paternal $\mathrm{H}-2 \mathrm{~K}^{\mathrm{b}}$ molecules expressed by the conceptus, a conclusion that is completely consistent with our OT-I studies showing a lack of direct recognition of fetal/placental OVA.

Maternal $\mathrm{CD}^{+} \mathrm{T}$ cells also recognize the conceptus exclusively by indirect antigen presentation. Our Act-mOVA mating system also allowed us to visualize maternal $\mathrm{CD} 4^{+} \mathrm{T}$ cell responses to the conceptus. Such responses have not previously been reported to our knowledge. For these experiments, we used $\mathrm{CD} 4^{+} \mathrm{T}$ cells isolated from OT-II TCR transgenic mice (25). These cells recognize the $\mathrm{OVA}_{323-339}$ epitope presented by the MHC class II molecule I- $\mathrm{A}^{\mathrm{b}}$. To map the spatial and temporal distribution of antigen presentation, we again used B6CBAF1 females as mating partners and adoptively transferred OT-II cells from mice that had been bred onto a B6CBAF1 background. We also extended the window of $\mathrm{T}$ cell exposure to a 4-day period to account for the less vigorous response of OT-II versus OT-I cells we observed for this strain background. Nonetheless, the overall pattern of response was very similar in the 2 cell types. Thus, OT-II proliferation in Act-mOVA-mated females was first evident at mid-gestation in the uterine LNs and spleen and became largely systemic by E15.5 (Figure 6A). Like OT-I and BM3 T cells, OT-II T cells were virtually undetectable within implantation sites ( $<5$ cells per implantation site per million cells injected; data not shown) and showed no obvious selective enrichment or depletion at E18.5 in $\mathrm{OVA}^{+}$versus OVA- implantation sites.

To determine the pathways of OVA presentation to OT-II cells, we mated Act-mOVA males to $\mathrm{B} 6$ congenic B6.C- $\mathrm{H} 2^{\text {bm } 12}$ mice, which bear a mutant I-A ${ }^{\mathrm{b}}$ molecule, and transferred OT-II $R A G 1^{-/-}$ 

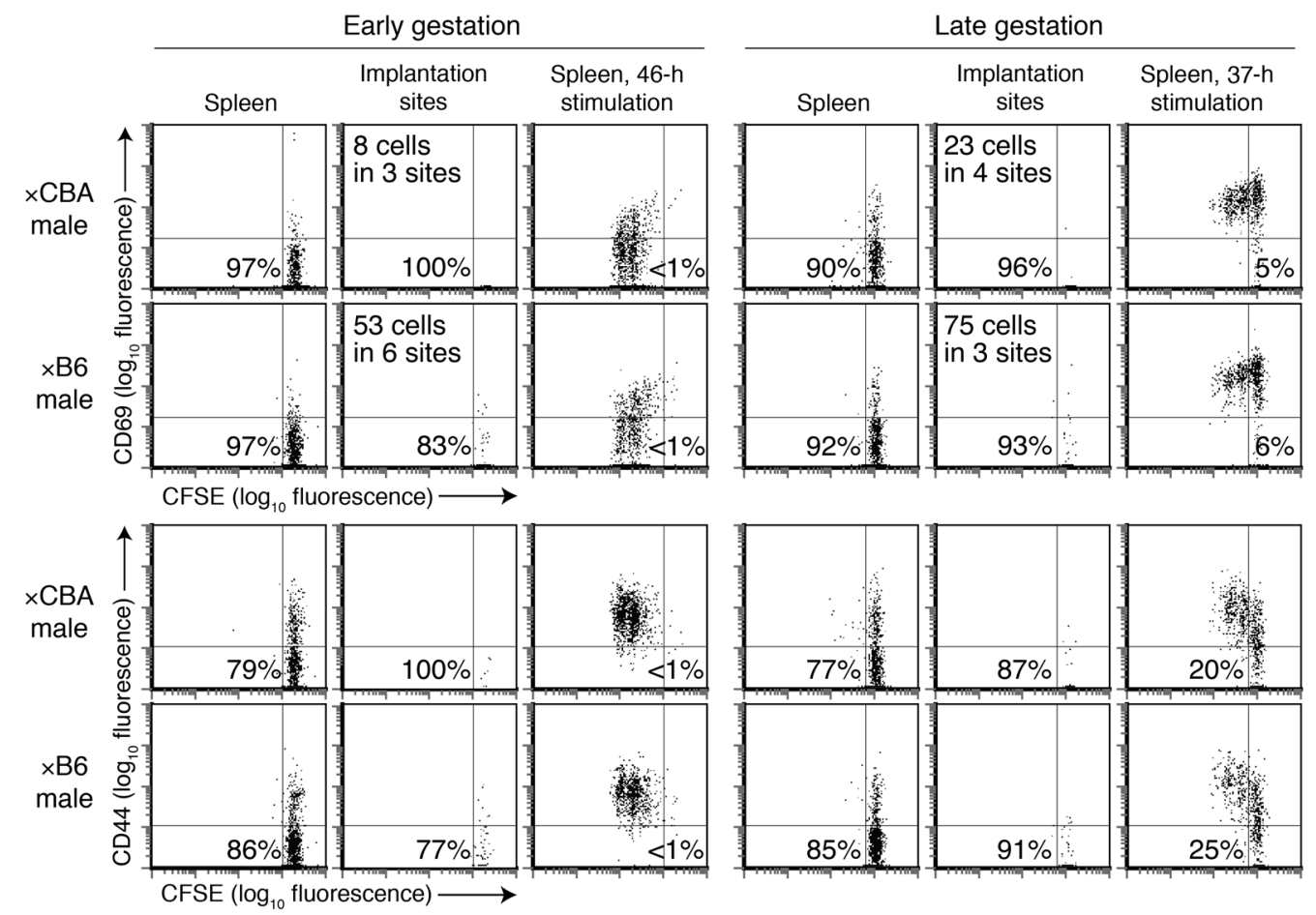

Figure 5

Maternal BM3 cells directly alloreactive with paternal $\mathrm{H}-2 \mathrm{~K}^{\mathrm{b}}$ show immune ignorance during gestation. CBA females mated to either CBA males or B6 males were adoptively transferred with CFSE-labeled BM3 T cells in during the first half of gestation (E5.5-E10.5). Splenocytes and implantation sites (pooled decidua/placentas) were analyzed by flow cytometry either 2 days later (early gestation, E7.5-E9.5) or 6-7 days later (late gestation, E13.5-E17.5). Percentages indicate the fraction of BM3 cells with CFSEundilutedCD69lo or CFSEundiluted CD44 ${ }^{10}$ naive phenotypes (cells in the lower-right quadrant). The total number of BM3 cells recovered from implantation sites is noted. Some mice were also stimulated by the intravenous injection of $5 \times 10^{6}$ B6CBAF1 splenocytes at the times indicated before sacrifice. Implantation sites are not shown for these mice since they showed a dramatic loss of BM3 cells from blood and implantation sites, likely due to their retention within secondary lymphoid organs. Data are representative of $n=4-7$ mice per group in 2 independent experiments. In additional experiments, $n=6$ CBA females mated to B6 males also failed to show evidence of TCR engagement in transferred BM3 cells when assayed for proliferation alone.

cells in late gestation. Unlike in the experiments with B6.C-H $2^{\mathrm{bm} 1}$ mice described above, there was no expectation of an alloresponse against the transferred cells, since they do not express MHC class II, and indeed their 4-day survival in B6 and B6.C-H2 $2^{b m 12}$ hosts was identical (data not shown). As seen in Figure 6B, OT-II cells transferred into Act-mOVA-mated B6.C-H2 ${ }^{\text {bm12 }}$ females showed no proliferative response, whereas a vigorous response was seen in ActmOVA-mated B6 females. These results demonstrate that OT-II cells, like OT-I cells, respond to OVA expressed by Act-mOVA concepti exclusively via indirect presentation.

Clonal deletion and defective priming of $\mathrm{CD}^{+} \mathrm{T}$ cells following exposure to fetal/placental OVA. Last, we used OT-I adoptive transfers to study the behavior of OVA-reactive $\mathrm{CD}^{+} \mathrm{T}$ cells in Act-mOVAmated females. Within 4 day after transfer at mid- to late gestation, OT-I cells had expanded 5-fold in uterine LNs and 3-fold in the spleen and subcutaneous LNs. However, the cells did not accumulate further, and at 3 weeks after delivery $(\sim 1$ month after transfer) were as prevalent as OT-I cells transferred into B6-mated females (Figure 7A). Since the 8 cell divisions we could visualize in Act-mOVA-mated females predicted a 256-fold expansion, their failure to accumulate strongly implied ongoing cell death. Such clonal deletion is frequently seen when $\mathrm{T}$ cells encounter antigen in the absence of inflammatory signals or under the influence of negative regulation $(27,31-34)$. For example, the injection of 100 $\mu \mathrm{g}$ endotoxin-free OVA into virgin females induced a maximal 2- to 4-fold expansion of OT-I cells after 3 days in all secondary lymphoid organs (Figure 7A), despite multiple rounds of division (e.g., see Figure 7C). In contrast, injection of virgin females with $100 \mu \mathrm{g}$ OVA plus agonistic anti-CD40 antibodies and the TLR3 ligand poly(I:C), an adjuvant regimen known to evoke strong antiOVA cellular immunity (35), induced a long-lasting 50- to 100-fold expansion of OT-I cells (Figure 7A).

Further experiments also revealed priming defects in OT-I cells when they were exposed to fetal/placental OVA. These defects were again consistent with generally accepted patterns of peripheral $\mathrm{T}$ cell tolerance $(27,31-34)$. First, splenic OT-I cells in Act-mOVAmated females failed to downregulate CD62 ligand (CD62L) and upregulate CD25, 2 changes in surface marker expression associated with productive immune responses (Figure 7B). Second, the cells expressed low levels of the effector cytokines IFN- $\gamma$, IL-2, and IL-4 (Figure 7C). We observed the same defective cytokine and activation expression profiles in the uterine and subcutaneous LNs (Figure 7D and data not shown), which argues against any regional regulation of the immune response. OT-I cells also expressed relatively low levels of IFN- $\gamma$ and IL- 2 when virgin hosts were injected with OVA in the absence of adjuvant but expressed high levels of IFN- $\gamma$ and IL- 2 when immunized with OVA plus anti-CD40 antibodies and poly(I:C) (Figure 7, C and D). Last, in vivo killing assays 


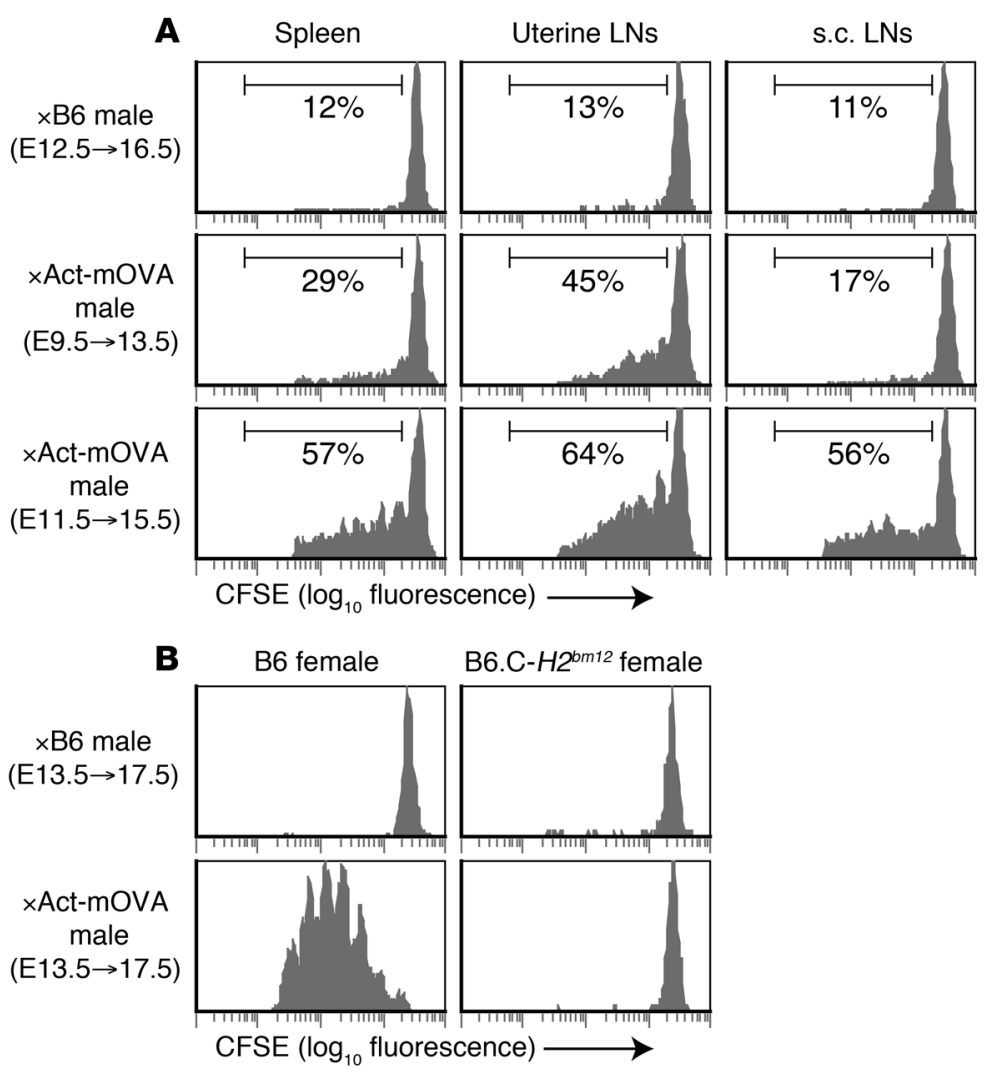

\section{Figure 6}

Maternal OT-II CD4+ T cells respond to fetal/placental OVA via indirect presentation. (A) OT-II cell proliferation in secondary lymphoid organs. B6CBAF1 females mated to ActmOVA or $B 6$ control males were adoptively transferred with CFSE-labeled B6CBAF1 OT-II T cells. OT-I cells were visualized by flow cytometry at the end of their indicated 4-day window of exposure. The percentage of cells undergoing more that 1 cell division is noted; such cells were only rarely observed in B6-mated females at any stage of gestation. Data are representative of $n=6$ Act-mOVA-mated mice and $n=6$ B6-mated mice over 3 independent experiments. (B) Indirect presentation of fetal/placental OVA. Splenocytes from B6- or Act-mOVA-mated B6 or B6.C-H2bm12 females were analyzed 4 days after the injection of CFSE-labeled OT-II cells late in gestation (on or after E13.5). Data are representative of 2 independent experiments, encompassing $n=5$ Act-mOVA-mated B6.C-H $2^{\mathrm{bm} 12}$ mice, $n=5$ Act-mOVAmated B6 mice, and $n=2-4$ mice each in the 2 other mating groups. Identical results were obtained with LN cells. performed 6 days after OT-I transfer detected only low levels of SIINFEKL-specific cytotoxicity in Act-mOVA-mated females as well as virgins injected with OVA without adjuvant, whereas virgins injected with OVA plus anti-CD40 antibodies and poly(I:C) showed strong cytotoxicity (Figure 8A).

As expected from the above results with OT-I adoptive transfers, B6-mated females, Act-mOVA-mated females, and OVAinjected virgin females all showed undetectable SIINFEKL-specific cytotoxicity when they bore unmanipulated $\mathrm{T}$ cell repertoires (Figure 8B, top row). Presumably, endogenous OVA-reactive T cells in Act-mOVA-mated females were undergoing the same abortive expansion and defective priming we observed for transferred OT-I cells. To test whether exogenous adjuvants could "convert" this abortive response into productive cellular immunity, we treated mice with anti-CD40 antibodies and poly(I:C) at mid-gestation (Figure 8B, bottom row). Interestingly, strong SIINFEKL-specific cytotoxicity could be detected in some Act-mOVA-mated females 6 days later (e.g., the mouse for which results are shown in Figure $8 \mathrm{~B})$; however, this response was not as robust on average as that seen with virgin females (Figure 8, B and C).

Consistent with our previous results showing that anti-CD40 antibody treatment alone does not induce abortion in either syngeneic or fully allogeneic mating combinations when given from E9.5 onward (26), treatment with anti-CD40 antibodies plus poly(I:C) in the above-described experiments did not induce wholesale abortion in B6- or Act-mOVA-mated females. Remarkably, however, we could not detect a specific loss of Act-mOVA concepti. In this set of mice, Act-mOVA ${ }^{+}$concepti represented 33 of 78 (42\%) of total concepti from untreated Act-mOVA-mated females and 17 of 44 (39\%) of total concepti from Act-mOVA-mated females treated with anti-CD40 antibodies and poly(I:C). Thus, the presence of documented anti-OVA CTL activity in pregnant females did not induce the death of Act-mOVA ${ }^{+}$concepti.

\section{Discussion}

A system to study maternal $T$ cell responses toward the allogeneic fetus. In the present study, we describe a system that enables the clear visualization of $\mathrm{CD}^{+}$and $\mathrm{CD} 8^{+} \mathrm{T}$ cell responses to the fetus. The system is based upon assessing the behavior of adoptively transferred anti-OVA TCR transgenic T cells in wild-type females impregnated by Act-mOVA transgenic males. The Act-mOVA transgene is thus targeted specifically to the fetus, with particularly high expression in trophoblast giant cells and endovascular trophoblasts directly at the maternal/fetal interface.

Strikingly, the hematogenous release of OVA from the conceptus induced the proliferation of both OT-I $\left(\mathrm{CD}^{+}\right)$and OT-II $\left(\mathrm{CD} 4^{+}\right)$ anti-OVA $T$ cells throughout all secondary maternal lymphoid organs, in parallel with the local accumulation of OVA on FDCs. Since FDCs are a nonmigratory cell type not thought to be involved in $\mathrm{T}$ cell antigen presentation, a likely scenario is that blood-borne OVA was ingested and processed by resident professional APCs. Initial high splenic uptake would explain the relatively delayed and variable presentation observed in the subcutaneous LNs. Conversely, the early and consistent appearance of OVA in the uterine LNs suggests additional transport via the regional lymphatics.

Two lines of evidence suggest that trophoblasts were the main if not sole source of shed antigen, as would be expected from straightforward topological considerations and the high OVA expression level of endovascular trophoblasts. Most important, we could rule out a substantial contribution from migratory embry- 
A
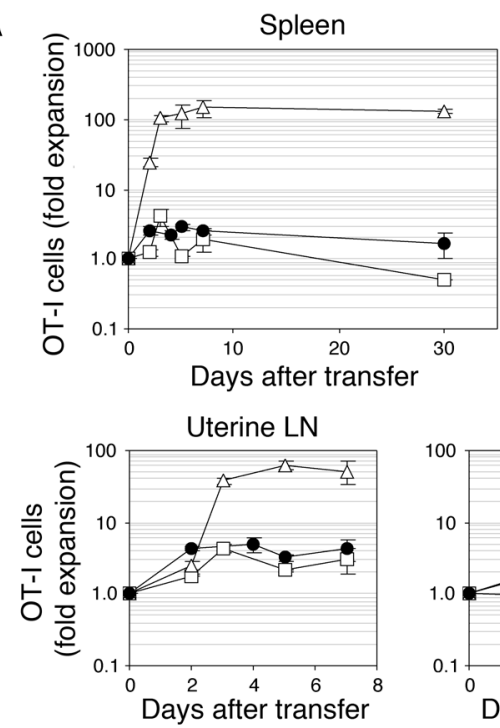

C
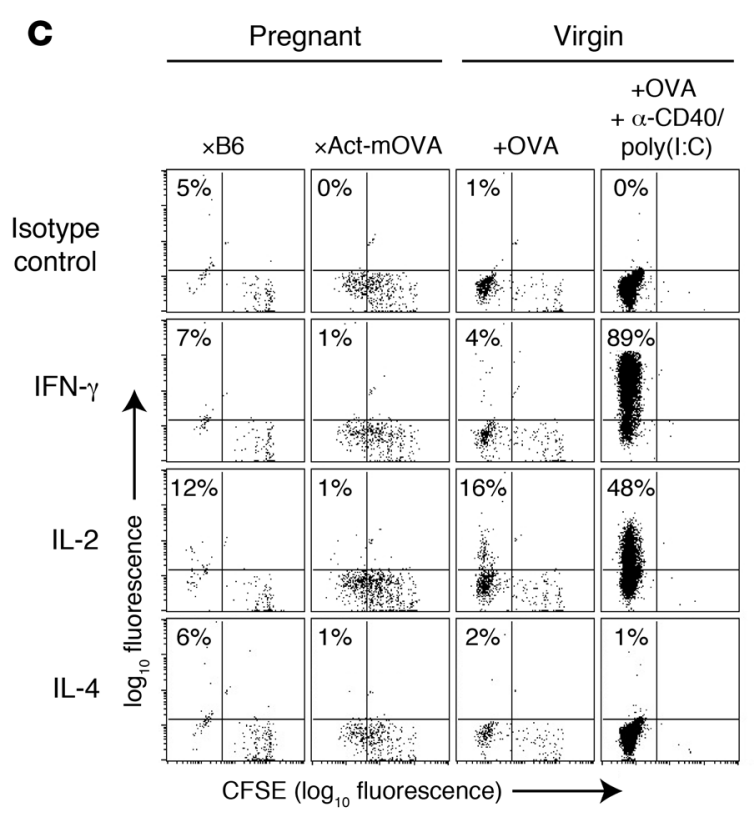

B
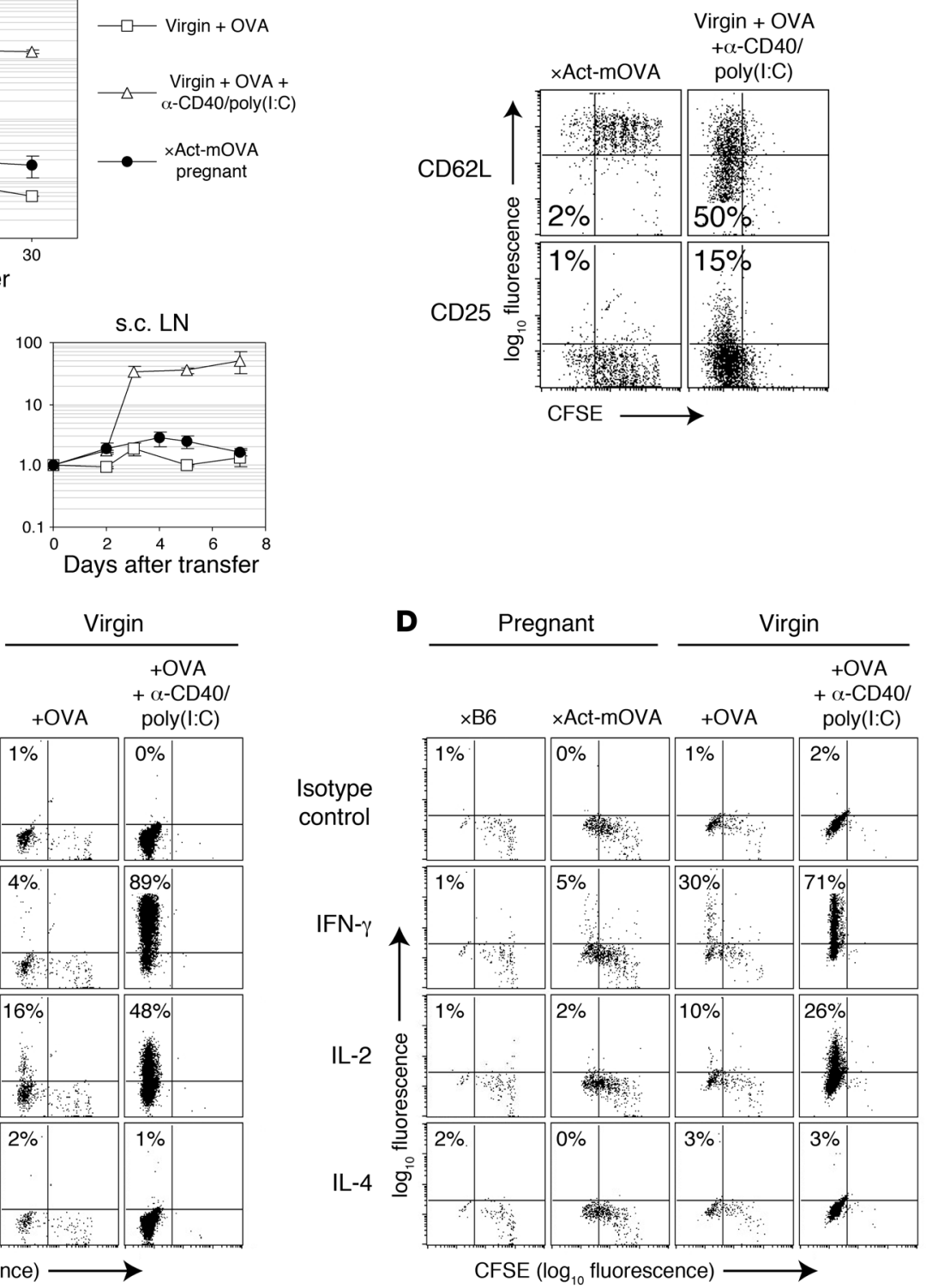

Figure 7

Defective OT-I cell priming in pregnant mice bearing Act-mOVA concepti. (A) Impaired cell accumulation. The number of OT-I cells remaining after transfer into Act-mOVA-mated females was calculated as a percentage of total CD8 ${ }^{+} \mathrm{T}$ cells, then normalized to the number of cells remaining after equivalent time periods in B6-mated mice injected in parallel. Similarly, the number of OT-I cells remaining in virgin females treated with OVA or OVA plus anti-CD40 antibodies and poly(I:C) was normalized to the number remaining in untreated virgins. Each point represents mean \pm SEM of $n=3-10$ Act-mOVA-mated or $n=2-3$ virgin mice. (B) Impaired modulation of CD62L and CD25 expression. OT-I cells were analyzed either 7 days after transfer into E10.5 Act-mOVA-mated or in virgin mice 3 days after treatment with OVA plus anti-CD40 antibodies and poly $(\mathrm{I}: \mathrm{C})$. Plots are representative of 3 independent experiments, each with individual mice or pools of $n=2-5$ Act-mOVA-mated and $n=1-3$ virgin mice per experiment. Plots display similar cell numbers in the 2 groups of mice, even though this does not represent their relative prevalence in vivo. The percentage of OT-I cells with altered marker expression is shown. (C and D) Impaired cytokine expression. Seven days after transfer into E10.5 pregnant mice, OT-I cells in the spleen (C) or uterine LNs (D) were stained for intracellular cytokines. Virgin females were similarly analyzed 7 days after indicated treatments given at the time of OT-I transfer. The percentage of OT-I cells with a CFSElo ${ }^{\circ}$ cytokine ${ }^{+}$phenotype is shown. Data are representative of 2 independent experiments, each with a pool of $n=4$ Act-mOVA-mated mice. The other treatment groups show data representative of individual mice or pools encompassing a total of $n=2-6$ mice per group.

onic hematopoietic cells, since we could not recapitulate the pattern of OVA accumulation on FDCs by intravenously injecting B6-mated females with unfractionated spleen cells (both rbc and wbc) from late-gestational Act-mOVA embryos or Act-mOVA neonates. A nonhematopoietic source of antigen is also consistent with previous work showing that nucleated fetal cells are very rare in the blood of pregnant mice (36). Second, our estimate for when OVA first becomes available for presentation in the spleen, E10.5, correlated with the invasion of OVA-expressing trophoblasts into the maternal arterioles of the decidua. Endovascular 
A
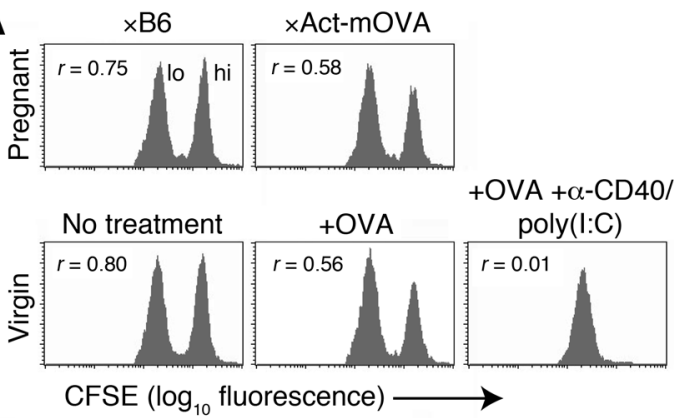

B

B Pregnant

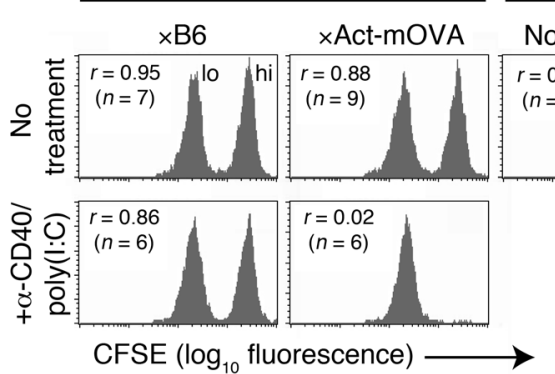

C

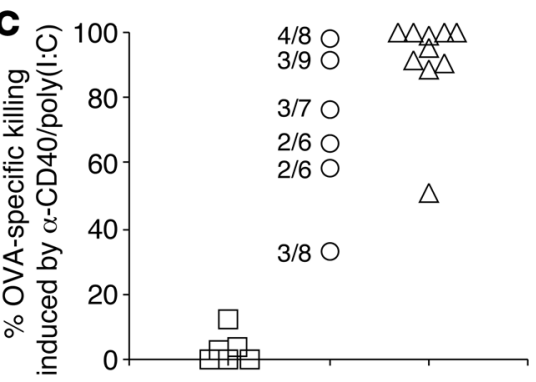

Virgin

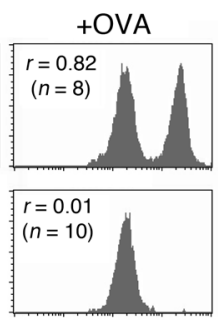

$\square \times \mathrm{B} 6$ pregnant

$\mathrm{O} \times$ Act-mOVA pregnant

$\triangle$ Virgin + OVA

\section{Figure 8}

Defective OVA-specific cytotoxic responses in Act-mOVA-mated mice. In vivo cytotoxicity assays were performed by injecting 50:50 mixtures of CFSEhi SIINFEKL-pulsed B6CBAF1 splenocytes (target cells) and CFSE ${ }^{\text {lo }}$ unpulsed splenocytes (control cells). The ratio ( $r$ ) was calculated as the number of CFSE ${ }^{\text {hi }}$ to $\mathrm{CFSE}^{\text {lo }}$ cells remaining 16 hours later; low ratios imply high levels of SIINFEKL-specific killing. (A) Low levels of SIINFEKLspecific cytotoxicity in Act-mOVA-mated mice receiving OT-I cells. E10.5-E11.5 pregnant or virgin females injected with OT-I cells were treated as indicated, then assayed 6 days later. Data are representative of 2 independent experiments, encompassing $n=4$ Act-mOVA-mated mice. (B) Absent SIINFEKL-specific cytotoxicity in Act-mOVA-mated mice with unmanipulated $T$ cell repertoires, unless treated with anti-CD40 antibodies and poly(I:C). E10.5-E13.5 pregnant or virgin females were treated as indicated, then assayed 6 days later. Data are from of 1 of 6 independent experiments. Each histogram shows representative data for a treatment group, except in the case of Act-mOVAmated females treated with anti-CD40 antibodies and poly(I:C), for which results for the mouse with the highest cytotoxicity are shown. The total number of mice in each group over all experiments is indicated. (C) CTL responses in Act-mOVA-mated females treated with CD40 antibodies and poly(I:C). The percentage of induced SIINFEKL-specific killing was calculated by normalizing the CFSE ${ }^{\text {hi }}$ to $\mathrm{CFSE}^{\mathrm{lo}}$ ratio for individual anti-CD40/ poly(I:C)-treated mice to the mean value of this ratio for their respective nonadjuvant-treated group. Act-mOVA-mated mice were assayed in 3 independent experiments; the number of ActmOVA concepti/total concepti for each of these mice is shown. invasion would thus expose the cell membranes of the cell population expressing the highest level of OVA in the midgestational conceptus directly to the maternal circulation. It is also possible that substantial contributions come from other trophoblast subtypes, particularly labyrinthine trophoblasts, which express lower levels of OVA on a per cell basis but have a very high contact surface area with maternal blood.

Unsurprisingly, the accumulation of OVA on FDCs could not be recapitulated by the intravenous injection of $1 \mathrm{mg}$ OVA into B6-mated B6CBAF1 females. Thus, OVA shedding from the conceptus is unlikely to have occurred through the simple extracellular cleavage and release of soluble monomers. One alternative is that OVA is released in association with apoptotic blebs formed by endovascular trophoblasts. A parallel process has already been described during human pregnancy in the release of syncytial knots from villous syncytiotrophoblasts (37). The binding of shed OVA to FDCs, a cell type notable for its ability to trap immune complexes and complement-fixed material (38), suggests potential pathways involved in the disposition of shed material.

Maternal T cells become aware of the fetus exclusively by the indirect pathway of transplant allorecognition. To determine the molecular pathways involved in OVA presentation, we used congenic female mating partners that were unable to present OVA-derived peptides to OT-I and OT-II cells. These pregnancies failed to show any OVAspecific proliferative responses, indicating a requirement for anti- gen uptake and presentation by maternal APCs. In other words, maternal T cells became aware of OVA expressed by the conceptus exclusively by indirect presentation.

In the case of MHC class II-restricted $\mathrm{T}$ cell responses, a requirement for indirect OVA presentation was not so surprising given the known lack of MHC class II expression by the murine placenta (14). In contrast, the requirement for indirect OVA presentation to maternal $C D 8^{+} \mathrm{T}$ cells (i.e., cross-presentation) was unexpected, since it suggested that $\mathrm{CD}^{+} \mathrm{T}$ cells in general were not able to directly engage fetal/placental MHC class I molecules. However, this was confirmed by our adoptive transfer experiments showing that BM3 cells directly alloreactive to $\mathrm{H}-2 \mathrm{~K}^{\mathrm{b}}$ remain ignorant of $\mathrm{H}-2 \mathrm{~K}^{\mathrm{b}}$ concepti.

Interestingly, our results with transferred BM3 cells are largely consistent with the minor phenotypic changes observed in a study by Zhou and Mellor that used intact BM3 transgenic mice as mating partners with $\mathrm{H}-2^{\mathrm{b}}(\mathrm{C} 57 \mathrm{BL} / 10)$ males (16). While originally taken as evidence for $\mathrm{T}$ cell recognition of paternal $\mathrm{H}-2 \mathrm{~K}^{\mathrm{b}}$, these changes represented only a $20 \%$ reduction in CD8 expression levels on splenic T cells and a 2 -fold increase in the number of $\mathrm{CD}^{+}$ cells. The cause of this increase was not determined, and proliferation was not directly assessed. Importantly, these authors noted that BM3 cells in females mated to $\mathrm{H}-2^{\mathrm{b}}$ males did not upregulate CD44, a sensitive marker of antigen exposure, and responded to ex vivo challenge as though they were naive. The minor discrepancies between our findings and those of Zhou and Mellor might 
thus simply reflect the use of intact TCR transgenic mice versus the adoptive transfer of TCR transgenic T cells. Overall, the data indicate that maternal T cells do not directly recognize MHC molecules expressed by the conceptus.

This conclusion strongly challenges the current dogma established by another study that addressed maternal $\mathrm{T}$ cell responses toward paternal MHC molecules (11). This study used TCR transgenic mice expressing the Des-TCR clonotype, which like the BM3 clonotype is alloreactive with $\mathrm{H}-2 \mathrm{~K}^{\mathrm{b}}$ (11). When intact Des-TCR transgenic females were mated to $\mathrm{H}-2^{\mathrm{b}}$ males, a large number of splenic $\mathrm{T}$ cells showed substantial phenotypic changes as early as E9.5. Such a response is clearly at odds with the otherwise undetectable level of MHC class I expression by the early conceptus, and here again cell proliferation was not directly assessed. Importantly, the opposing response seen with the Des-TCR versus the BM3 system cannot be explained by a differential sensitivity of the Des-TCR versus BM3 clonotypes, as both are considered high-avidity TCRs (39). If anything, Des-TCR T cells are more difficult to activate than BM3 T cells, since they require CD8 coreceptor engagement in addition to TCR engagement (39). Instead, the results with DesTCR T cells might relate to their atypical behavior (dramatic CD8 and TCR downregulation upon activation, which we did not see in any of our adoptive transfer experiments; Supplemental Figure 4 and data not shown).

Limited pathways of fetal allorecognition and mechanisms of fetomaternal tolerance. An absence of direct allorecognition of the fetus has major implications for mechanisms of fetomaternal tolerance. Direct allorecognition is thought to occur with all transplanted solid organs, and while its importance has best been established for acute cardiac transplant rejection (4-8), direct allorecognition is thought to provide a primary stimulus for acute rejection in general (1). Indeed, directly alloreactive $T$ cells outnumber indirectly reactive $T$ cells by a greater than 10 to 1 margin (2) and in absolute terms have recently been estimated to constitute approximately $0.5 \%-7 \%$ of the peripheral $\mathrm{T}$ cell repertoire $(2,3)$. Thus, the lack of direct recognition of the fetal allograft removes a major immunological threat to fetal survival. The reason for this substantial level of immune ignorance is currently unclear but may involve the lack of a substantial "passenger leukocyte" population or professional APC population of fetal origin within the placenta and the virtually undetectable levels of MHC class I expression by the conceptus during the first half of gestation.

The absence of direct allorecognition, however, does not remove all immunological danger for the allogeneic fetus. Indirect presentation has become increasingly appreciated as an important adjunct pathway in transplant allorecognition and in the case of skin grafts is sufficient to mediate rejection in the absence of direct presentation $(5,7,40-43)$. Our results suggest that 2 additional mechanisms may therefore be important for insuring fetomaternal tolerance. First, we find that $\mathrm{T}$ cells indirectly reactive to fetal/placental OVA undergo clonal deletion and are poorly primed during pregnancy. This kind of response, while consistent with many general studies of peripheral $\mathrm{T}$ cell tolerance, would not have been expected based on the transplantation literature. Rather, indirectly alloreactive $T$ cells can be primed to both skin and heart transplants, even in experimental situations where direct allorecognition is absent $(5,7,40-44)$. One possibility for the differential behavior of the fetus versus surgical organ transplants is that pregnancy may lack the local and/or systemic inflammatory response associated with surgery. However, the reduced ability of
anti-CD40 antibodies and poly(I:C) to induce a SIINFEKL-specific cytotoxicity in Act-mOVA-mated females suggests that negative regulation might also play a role in poor $\mathrm{T}$ cell priming. This would be in line with recent studies suggesting that regulatory $\mathrm{T}$ cells and the inhibitory costimulatory molecule PDL1 are involved in fetomaternal tolerance $(21,22)$. It is also possible that pregnancy hormones or factors released from the conceptus in association with OVA itself have dominant regulatory function.

The second mechanism that may insure fetomaternal tolerance is an inability of effector $T$ cells to attack the fetus. Thus, we find that anti-OVA CD8 ${ }^{+} \mathrm{T}$ cells artificially primed during pregnancy by anti-CD40 antibodies and poly(I:C) are unable to kill Act-mOVA ${ }^{+}$ concepti. Although we do not currently know whether placental cells express sufficient $\mathrm{K}^{\mathrm{b}}$ /SIINFEKL complexes to be recognized by effector $\mathrm{CD}^{+} \mathrm{T}$ cells, this result is reminiscent of the poor immunological assault encountered by heart transplants when recognized exclusively by indirectly alloreactive, yet primed, $\mathrm{CD}^{+}$ $\mathrm{T}$ cells (7). This latter phenomenon has been linked to the fact that heart transplants largely retain donor endothelial cells to line their blood vessels (7). In contrast, the rejection of skin grafts by indirectly alloreactive $\mathrm{CD}^{+} \mathrm{T}$ cells has been suggested to be due to their revascularization by host endothelial cells and the potential for these cells to locally cross-present antigen (7). Interestingly, the placenta resembles a cardiac allograft in that the cells (i.e., trophoblasts) in direct contact with blood are of donor origin.

In summary, our results have revealed 3 independent mechanisms that simultaneously protect the fetus from rejection by the maternal immune system. First, there is an absence of direct recognition of the fetus, which means that the large number of directly alloreactive $T$ cells that typically drive acute transplant rejection are simply ignorant of the fetal allograft. Second, those maternal $\mathrm{T}$ cells that can indirectly recognize the fetus are poorly primed and instead undergo clonal deletion. Third, indirectly alloreactive $\mathrm{T}$ cells, if spuriously (or experimentally) activated, are still unable to attack the fetus. Each of these levels of regulation in turn raises its own set of mechanistic questions.

Limited pathways of fetal allorecognition and mechanisms of early pregnancy loss. Besides the absence of direct recognition of the fetus over the entirety of gestation, several lines of evidence suggest that indirect recognition of the early embryo may be severely limited as well. First, presentation of fetal/placental OVA commenced no earlier than E10.5, despite expression by trophoblasts at the forefront of the ectoplacental cone as early as E7.5. Second, maternal T cells are ignorant of $\mathrm{H}-\mathrm{Y}$ antigen from the time of copulation up until E6.5, which covers the period of semen exposure as well as early postimplantation development (19). Third, the virtual absence of MHC expression by the early conceptus would in itself predict limited possibilities for indirect recognition, since peptides derived from donor MHC molecules are thought to be the primary source of minor antigens for the indirect recognition of surgical organ transplants (2). While it is possible that other fetal or placental minor histocompatibility antigens have higher expression levels than OVA or H-Y antigen, or increased accessibility to maternal APCs, current evidence thus suggests that maternal T cells may be largely ignorant of the fetus early in gestation.

This interpretation is clearly discordant with the increasing number of studies that show the induction of early pregnancy loss in an allospecific fashion, i.e., in allogeneic but not syngeneic mating combinations. These studies show fetal damage as early as E9.5-E13.5 (20-23), which is remarkably fast considering that implantation 
occurs late on E3.5. For comparison, fully mismatched wild-type skin grafts, recognized by both direct and indirect antigen presentation, are rejected after about 10 days. Thus, the question arises: In the case of allospecific early pregnancy loss, how has the maternal immune system discriminated self from non-self?

The resolution of this paradox will not only be important for our basic understanding of the immunology of pregnancy but will have clinical implications for organ transplantation and early pregnancy failure in humans. One possibility is that nonspecific, but nonlethal, damage to the early embryo induces embryonic expression of MHC molecules or potent minor histocompatibility antigens and that $\mathrm{T}$ cell activation and ultimate fetal demise are secondary events. Another possibility invokes a role for NK cells. These cells are by far the most abundant leukocyte population at the maternal/fetal interface at the time of early embryo loss and have recently been implicated in the rejection of solid organs (45). Furthermore, NK cells can be activated by stress-induced ligands rather than simply the absence of self-MHC (46). It is therefore plausible that their behavior at the maternal/fetal interface might be regulated by developmental processes intrinsic to the strain combination, rather than by antigenic disparities between father and mother. Indeed, recent work in humans has suggested that decidual NK cells engage non-MHC class I ligands at the mater$\mathrm{nal} /$ fetal interface (47). Intriguingly, a model of cardiac allograft vasculopathy was recently shown to require a collaboration between NK cells and $\mathrm{CD}^{+} \mathrm{T}$ cells that had neither direct nor indirect alloreactivity toward donor antigens (48). This suggests that bystander $\mathrm{T}$ cell activation might be an important mechanism for NK cell-mediated allograft damage and might explain why manipulations that cause allospecific abortion often require intact adaptive immune systems. The inflammatory cytokines produced by the NK-T cell collaboration might cause fetal demise by interfering with developmental processes at the maternal/fetal interface, by causing local derangements in hemostasis, or by inhibiting ovarian progesterone biosynthesis if produced at sufficient systemic levels (26).

\section{Methods}

Mice. Act-mOVA and BM3 mice were gifts of M. Jenkins (University of Minnesota Medical School, Minneapolis, Minnesota, USA) and A. Mellor (Medical College of Georgia, Augusta, Georgia, USA), respectively. B6CBAF1, B6.C$H 2^{b m 1}$, B6.C- $H 2^{b m 12}$, and CBA/J (CBA) mice were obtained from The Jackson Laboratory. B6, OT-II $R A G 1^{-/-}$, and $H 2 K^{b} D^{b}$ knockout mice were obtained from Taconic. Homozygous OT-I RAG2-/- CD45.1/1 mice, otherwise on a B6 background, were provided by C. Benoist and D. Mathis (Harvard Medical School) and were mated to CBA mice to generate OT-I CD45.1/2 B6CBAF1 cell donors. The CD45.1 congenic marker was used for OT-I identification when the cells were expected to become CFSEdilute. OT-II mice were mated to CBA mice to generate OT-II B6CBAF1 cell donors. Mice were maintained in specific pathogen-free facilities, and all experiments were approved by the Standing Committee on Animals of Harvard Medical School and New York University School of Medicine. Noon of the day of the appearance of the copulation plug was counted as E0.5. Some females were primed with $2 \mathrm{IU}$ pregnant mare serum gonadotropin (Sigma-Aldrich) and 5 IU human chorionic gonadotropin (Sigma-Aldrich) to ensure synchronous mating (experiments with $H 2 K^{b} D^{b}$ knockout mice and B6.C-H $2^{b m 12}$ mice). All females referred to as being "Act-mOVA-mated" either had clear immunological or immunohistochemical evidence of bearing OVA concepti or had their embryos or pups PCR genotyped with the primers $5^{\prime}$-TTATTCGTTCAGCCTTGCCAGTAG-3' and 5'-GCTCCAGGATCTTCATTTTCTCAG-3'.
Adoptive transfers, flow cytometry, and in vivo killing assays. Donor cells were isolated from the spleen and all peripheral LNs, purified by negative selection using magnetic beads coated with antibodies against CD19, DX5, CD11b, CD11c, MHC class II, B220, and either CD4 (for OT-I and BM3 purification) or CD8 (for OT-II purification) as per the manufacturer's instructions (Miltenyi Biotec), then labeled with $5 \mu \mathrm{M}$ CFSE (Molecular Probes; Invitrogen) for 10 minutes at $37^{\circ} \mathrm{C}$ in PBS. $0.5 \times 10^{6}$ to $4 \times 10^{6}$ cells per mouse were injected via the tail vein. Female donors were used for all experiments involving cell recovery later than 10 days after transfer. Some mice additionally received intravenous injections of $5 \times 10^{6}$ B6CBAF1 splenocytes with or without prior loading with $50 \mu \mathrm{g} / \mathrm{ml}$ SIINFEKL peptide (Sigma-Aldrich) for 1 hour in PBS at $37^{\circ} \mathrm{C}$. OVA, anti-CD 40 antibodies (clone FGK45.5; prepared by Bio Express), and poly(I:C) (Sigma-Aldrich) were given by intraperitoneal injection at doses of $100 \mu \mathrm{g}, 100 \mu \mathrm{g}$, and $50 \mu \mathrm{g}$, respectively, except in a subset of the in vivo killing assays performed on mice containing unmanipulated $\mathrm{T}$ cell repertoires, where OVA was given at a dose of $500 \mu \mathrm{g}$. Endotoxin was removed from OVA (Grade VI; Sigma-Aldrich) with Detoxi-Gel (Pierce), and remaining levels were found to be less than 0.06 endotoxin units per injection as determined by the LAL assay (BioWhittaker).

After sacrifice, splenocytes were isolated by mechanical disaggregation followed by rbc lysis, whereas LN cells and cells from implantation sites were isolated by digestion in $2 \mathrm{mg} / \mathrm{ml}$ collagenase $\mathrm{D} / 0.2 \mathrm{mg} / \mathrm{ml}$ dispase $\mathrm{II} / 30 \mu \mathrm{g} / \mathrm{ml}$ DNAase I or in 0.28 Wunsch units Blendzyme 3/DNAase I, all purchased from Roche Applied Science. Subcutaneous LN preparations contained the inguinal, brachial, axillary, and superficial cervical nodes. When blood and implantation sites were included in the analysis, mice were heparinized prior to sacrifice. The decidua and placenta were separated from the embryo, yolk sac, and myometrium and stored on ice, while embryonic genotypes were rapidly determined by real-time PCR, when necessary. Genotype-equivalent tissues were then pooled, minced, and digested in 0.28 Wunsch units Blendzyme 3/DNAase I prior to flow cytometry. A similar protocol was used to prepare embryonic and neonatal Act-mOVA spleen cells for intravenous injection.

Flow cytometry was performed on cells pretreated with the FcyR-blocking $\mathrm{mAb} 2.4 \mathrm{G} 2$ and using antibodies purchased from BD Biosciences and BioLegend. We used either a 2-laser FACSCalibur cytometer (BD Biosciences) and CellQuest Pro software for analysis (version 5.2.1; BD Biosciences) or a 3-laser LSR II cytometer (BD Biosciences) and FCS Express V3 software for analysis (version 3.00.0311; De Novo Software). Aside from their CFSE label, transferred OT-I cells were identified with various combinations of APC- or PE-Cy7-conjugated anti-CD8 $\alpha$, PE-conjugated anti-V $\alpha 2$, and biotin-conjugated anti-CD45.1/streptavidin-PerCP-Cy5. Transferred OT-II cells were similarly identified with anti-V $\alpha 2$ and APC- or PE-Cy7-conjugated anti-CD4 and BM3 cells with anti-CD8 $\alpha$ and PE-conjugated anti-TCR- $\beta$. PE-conjugated anti-CD62L and anti-CD25, APC-conjugated anti-CD69, and Pacific blue-conjugated anti-CD44 were used for cell-surface marker expression studies. Intracellular cytokine expression was performed as previously described using PE-conjugated antibodies (49) after the splenocytes or LN cells were cultured for 4 hours in media containing $1 \mu \mathrm{M}$ SIINFEKL peptide, with $3 \mu \mathrm{M}$ monensin added for the last 2 hours. In vivo killing assays were performed as previously described (35); target cells were injected 6 days after immunization, and mice were sacrificed 16 hours later.

Immunohistochemistry. For OVA and CD35 staining, tissues were fixed overnight at $4{ }^{\circ} \mathrm{C}$ in $4 \%$ paraformaldehyde prior to routine paraffin embedding. Following dewaxing, $5-\mu \mathrm{m}$ tissue sections were treated with $3 \% \mathrm{H}_{2} \mathrm{O}_{2}$ in methanol for 20 minutes, then with $1 \mathrm{mg} / \mathrm{ml}$ trypsin (prepared freshly from tablets; Sigma-Aldrich) for 10 minutes at $37^{\circ} \mathrm{C}$, and then blocked in $1 \% \mathrm{BSA} / 3 \%$ rabbit serum $/ 3 \%$ donkey serum $/ 0.4 \%$ Triton $\mathrm{X}-100$. Washes were in PBS containing $0.01 \%$ Triton X-100, and sections were counterstained with DAPI (Sigma-Aldrich) prior to digital photography. For anti- 
OVA immunostaining, sections were incubated overnight at $4^{\circ} \mathrm{C}$ with a 1:3,000 dilution of biotinylated rabbit anti-OVA antibodies (Research Diagnostics Inc.) in TNB buffer (PerkinElmer). HRP-conjugated streptavidin was then applied as the secondary reagent (PerkinElmer), followed by biotin-tyramide amplification (PerkinElmer) and incubation with streptavidin-Alexa Fluor 594 (Molecular Probes; Invitrogen) as the final fluorochrome. For double staining with anti-CD35 antibodies, a 1:1,000 dilution of rat anti-CD35 (clone 8C12; BD Biosciences) was included with the anti-OVA primary incubation. After tyramide amplification of the OVA signal as described above, peroxidase activity was quenched again with $3 \%$ $\mathrm{H}_{2} \mathrm{O}_{2}$ plus $0.1 \%$ sodium azide in PBS for 20 minutes at room temperature. Multiple-species preabsorbed HRP-conjugated donkey anti-rat antibodies (Jackson ImmunoResearch Laboratories) were then applied at a 1:200 dilution in TNB buffer, followed by FITC-tyramide amplification (PerkinElmer). Last, the sections were incubated with Alexa Fluor 594-conjugated streptavidin and Alexa Fluor 488-conjugated rabbit anti-FITC (Molecular Probes; Invitrogen). Rigorous controls involving the entire double staining protocol but solely omitting either one or the other primary antibody confirmed that each final signal was specific for the intended antigen. The high background fluorescence of rbc was an artifact of the tyramide amplification. Image processing was performed with Adobe Photoshop, including the tiling of $\times 4$ photomicrographs of entire implantation sites.

For MHC class I staining, tissues were fixed overnight at $4^{\circ} \mathrm{C}$ in $4 \%$ paraformaldehyde, equilibrated in $30 \%$ sucrose at $4^{\circ} \mathrm{C}$, embedded in OCT compound (Tissue-Tek; Sakura Finetek), and sectioned at $5 \mu \mathrm{m}$. Sections were immersed in acetone for 5 minutes at room temperature, air-dried for
10 minutes, treated with $3 \% \mathrm{H}_{2} \mathrm{O}_{2}$ in methanol for 20 minutes, then blocked in $1 \% \mathrm{BSA} / 3 \%$ goat serum $/ 0.4 \%$ Triton $\mathrm{X}-100$. Sections were then incubated overnight at $4{ }^{\circ} \mathrm{C}$ with a 1:300 dilution of rat anti-mouse MHC class I (clone ER-HR52; Abcam), followed by biotinylated goat anti-rat antibodies (1:400 dilution; Jackson ImmunoResearch Laboratories), HRP-conjugated streptavidin, and biotin-tyramide amplification. Streptavidin-Alexa Fluor 594 was used as the final fluorochrome. The tissues shown in the Supplemental Figure 3 were processed and stained at exactly the same time.

\section{Acknowledgments}

We are grateful to M. Jenkins, A. Mellor, C. Benoist, D. Mathis, and J. Iacomini for providing mice and thank A. Daggett for technical assistance; L. Kangaloo and L. de Elizalde for administrative support; M. Carroll, R. Obst, M. Townsend, M. Wein, and A. Aliprantis for valuable discussions; and J. Skok for critical reading of the manuscript. This work was supported by NIH grants RO1AI54370 to L.H. Glimcher and RO1-AI062980 to A. Erlebacher.

Received for publication February 13, 2006, and accepted in revised form February 20, 2007.

Address correspondence to: Adrian Erlebacher, Department of Pathology, New York University School of Medicine, Smilow Research Center, Room 311, 550 First Avenue, New York, New York 10016, USA. Phone: (212) 263-8926; Fax: (212) 263-8211; E-mail: adrian.erlebacher@med.nyu.edu.
1. Game, D.S., and Lechler, R.I. 2002. Pathways of allorecognition: implications for transplantation tolerance. Transpl. Immunol. 10:101-108.

2. Benichou, G., Valujskikh, A., and Heeger, P.S. 1999. Contributions of direct and indirect $\mathrm{T}$ cell alloreactivity during allograft rejection in mice. J. Immunol. 162:352-358.

3. Suchin, E.J., et al. 2001. Quantifying the frequency of alloreactive $T$ cells in vivo: new answers to an old question. J. Immunol. 166:973-981.

4. Campos, L., et al. 1995. Survival of MHC-deficient mouse heterotopic cardiac allografts. Transplantation. 59:187-191.

5. Chen, Y., Demir, Y., Valujskikh, A., and Heeger, P.S. 2003. The male minor transplantation antigen preferentially activates recipient CD4+ T cells through the indirect presentation pathway in vivo. J. Immunol. 171:6510-6518.

6. Pietra, B.A., Wiseman, A., Bolwerk, A., Rizeq, M., and Gill, R.G. 2000. CD4 T cell-mediated cardiac allograft rejection requires donor but not host MHC class II. J. Clin. Invest. 106:1003-1010.

7. Valujskikh, A., Lantz, O., Celli, S., Matzinger, P., and Heeger, P.S. 2002. Cross-primed CD8(+) T cells mediate graft rejection via a distinct effector pathway. Nat. Immunol. 3:844-851.

8. Grazia, T.J., et al. 2004. A two-step model of acute CD4 T-cell mediated cardiac allograft rejection. J. Immunol. 172:7451-7458.

9. Moffett, A., and Loke, C. 2006. Immunology of placentation in eutherian mammals. Nat. Rev. Immunol. 6:584-594.

10. Trowsdale, J., and Betz, A.G. 2006. Mother's little helpers: mechanisms of maternal-fetal tolerance. Nat. Immunol. 7:241-246.

11. Tafuri, A., Alferink, J., Moller, P., Hammerling, G.J., and Arnold, B. 1995. T cell awareness of paternal alloantigens during pregnancy. Science. 270:630-633.

12. Jaffe, L., Jeannotte, L., Bikoff, E.K., and Robertson, E.J. 1990. Analysis of beta 2-microglobulin gene expression in the developing mouse embryo and placenta. J. Immunol. 145:3474-3482.
13. Jaffe, L., Robertson, E.J., and Bikoff, E.K. 1991. Distinct patterns of expression of MHC class I and beta 2-microglobulin transcripts at early stages of mouse development. J. Immunol. 147:2740-2749.

14. Mattsson, R., Mattsson, A., Holmdahl, R., Scheynius, A., and Van der Meide, P.H. 1992. In vivo treatment with interferon-gamma during early pregnancy in mice induces strong expression of major histocompatibility complex class I and II molecules in uterus and decidua but not in extraembryonic tissues. Biol. Reprod. 46:1176-1186.

15. Rogers, A.M., Boime, I., Connolly, J., Cook, J.R., and Russell, J.H. 1998. Maternal-fetal tolerance is maintained despite transgene-driven trophoblast expression of MHC class I, and defects in Fas and its ligand. Eur. J. Immunol. 28:3479-3487.

16. Zhou, M., and Mellor, A.L. 1998. Expanded cohorts of maternal CD8+ T-cells specific for paternal MHC class I accumulate during pregnancy. J. Reprod. Immunol. 40:47-62.

17. Vacchio, M.S., and Hodes, R.J. 2003. CD28 costimulation is required for in vivo induction of peripheral tolerance in CD8 T cells. J. Exp. Med. 197:19-26.

18. Kearney, E.R., Pape, K.A., Loh, D.Y., and Jenkins, M.K. 1994. Visualization of peptide-specific T cell immunity and peripheral tolerance induction in vivo. Immunity. 1:327-339.

19. Seavey, M.M., and Mosmann, T.R. 2006. Paternal antigen-bearing cells transferred during insemination do not stimulate anti-paternal CD8+ T cells: role of estradiol in locally inhibiting CD8 $+\mathrm{T}$ cell responses. J. Immunol. 177:7567-7578.

20. Munn, D.H., et al. 1998. Prevention of allogeneic fetal rejection by tryptophan catabolism. Science. 281:1191-1193.

21. Aluvihare, V.R., Kallikourdis, M., and Betz, A.G. 2004. Regulatory $\mathrm{T}$ cells mediate maternal tolerance to the fetus. Nat. Immunol. 5:266-271.

22. Guleria, I., et al. 2005. A critical role for the programmed death ligand 1 in fetomaternal tolerance. J. Exp. Med. 202:231-237.

23. Duclos, A.J., Pomerantz, D.K., and Baines, M.G. 1994. Relationship between decidual leukocyte infiltration and spontaneous abortion in a murine model of early fetal resorption. Cell. Immunol. 159:184-193.

24. Ehst, B.D., Ingulli, E., and Jenkins, M.K. 2003. Development of a novel transgenic mouse for the study of interactions between CD4 and CD8 T cells during graft rejection. Am. J. Transplant. 3:1355-1362.

25. Barnden, M.J., Allison, J., Heath, W.R., and Carbone, F.R. 1998. Defective TCR expression in transgenic mice constructed using cDNA-based alpha- and beta-chain genes under the control of heterologous regulatory elements. Immunol. Cell Biol. 76:34-40.

26. Erlebacher, A., Zhang, D., Parlow, A.F., and Glimcher, L.H. 2004. Ovarian insufficiency and early pregnancy loss induced by activation of the innate immune system. J. Clin. Invest. 114:39-48. doi:10.1172/JCI200420645.

27. Kurts, C., Kosaka, H., Carbone, F.R., Miller, J.F., and Heath, W.R. 1997. Class I-restricted crosspresentation of exogenous self-antigens leads to deletion of autoreactive CD8(+) T cells. J. Exp. Med. 186:239-245.

28. Jiang, S., Herrera, O., and Lechler, R.I. 2004. New spectrum of allorecognition pathways: implications for graft rejection and transplantation tolerance. Curr. Opin. Immunol. 16:550-557.

29. Nikolic-Zugic, J., and Bevan, M.J. 1990. Role of selfpeptides in positively selecting the T-cell repertoire. Nature. 344:65-67.

30. Sponaas, A.M., et al. 1994. Induction of tolerance to self MHC class I molecules expressed under the control of milk protein or beta-globin gene promoters. Int. Immunol. 6:277-287.

31. Hernandez, J., Aung, S., Redmond, W.L., and Sherman, L.A. 2001. Phenotypic and functional analysis of CD8(+) T cells undergoing peripheral deletion in response to cross-presentation of self-antigen. J. Exp. Med. 194:707-717.

32. Quezada, S.A., et al. 2005. Analysis of the underlying cellular mechanisms of anti-CD154-induced graft tolerance: the interplay of clonal anergy and immune regulation. J. Immunol. 175:771-779.

33. Reinhardt, R.L., Khoruts, A., Merica, R., Zell, T., 
and Jenkins, M.K. 2001. Visualizing the generation of memory CD4 T cells in the whole body. Nature. 410:101-105.

34. Bonifaz, L., et al. 2002. Efficient targeting of protein antigen to the dendritic cell receptor DEC-205 in the steady state leads to antigen presentation on major histocompatibility complex class I products and peripheral CD8+ T cell tolerance. J. Exp. Med. 196:1627-1638.

35. Ahonen, C.L., et al. 2004. Combined TLR and CD40 triggering induces potent CD8 $+\mathrm{T}$ cell expansion with variable dependence on type I IFN. J. Exp. Med. 199:775-784.

36. Bonney, E.A., and Matzinger, P. 1997. The maternal immune system's interaction with circulating fetal cells. J. Immunol. 158:40-47.

37. Huppertz, B., and Herrler, A. 2005. Regulation of proliferation and apoptosis during development of the preimplantation embryo and the placenta. Birth Defects Res. C Embryo Today. 75:249-261.
38. Tew, J.G., et al. 1997. Follicular dendritic cells and presentation of antigen and costimulatory signals to B cells. Immunol. Rev. 156:39-52.

39. Auphan, N., et al. 1994. The degree of CD8 dependence of cytolytic T cell precursors is determined by the nature of the T cell receptor (TCR) and influences negative selection in TCR-transgenic mice. Eur. J. Immunol. 24:1572-1577.

40. Baratin, M., Bonin, K., and Daniel, C. 2004. Frontline: peripheral priming of alloreactive $\mathrm{T}$ cells by the direct pathway of allorecognition. Eur. J. Immunol. 34:3305-3314.

41. Braun, M.Y., et al. 2001. Acute rejection in the absence of cognate recognition of allograft by T cells. J. Immunol. 166:4879-4883.

42. He, C., and Heeger, P.S. 2004. CD8 T cells can reject major histocompatibility complex class I-deficient skin allografts. Am. J. Transplant. 4:698-704.

43. Sandner, S.E., et al. 2003. New TCR transgenic model for tracking allospecific CD4 T-cell activation and tolerance in vivo. Am. J. Transplant. 3:1242-1250.

44. Valujskikh, A., Zhang, Q., and Heeger, P.S. 2006. CD8 $T$ cells specific for a donor-derived, selfrestricted transplant antigen are nonpathogenic bystanders after vascularized heart transplantation in mice. J. Immunol. 176:2190-2196.

45. Maier, S., et al. 2001. Inhibition of natural killer cells results in acceptance of cardiac allografts in CD28 $8^{-/-}$mice. Nat. Med. 7:557-562.

46. Lanier, L.L. 2005. NK cell recognition. Annu. Rev. Immunol. 23:225-274.

47. Hanna, J., et al. 2006. Decidual NK cells regulate key developmental processes at the human fetalmaternal interface. Nat. Med. 12:1065-1074.

48. Uehara, S., et al. 2005. NK cells can trigger allograft vasculopathy: the role of hybrid resistance in solid organ allografts. J. Immunol. 175:3424-3430.

49. Ouyang, W., et al. 1998. Inhibition of Th1 development mediated by GATA-3 through an IL-4-independent mechanism. Immunity. 9:745-755. 